\title{
COUNTERGRADIENT FLUXES OF CONSERVED VARIABLES IN THE CLEAR CONVECTIVE AND STRATOCUMULUS-TOPPED BOUNDARY LAYER: THE ROLE OF THE ENTRAINMENT FLUX
}

\author{
STEPHAN R. DE ROODE \\ Institute for Marine and Atmospheric Research Utrecht (IMAU), Utrecht University, The \\ Netherlands \\ HARM J. J. JONKER \\ Thermofluids Section, Department of Applied Physics, Delft University of Technology, The \\ Netherlands \\ PETER G. DUYNKERKE ${ }^{\dagger}$ \\ Institute for Marine and Atmospheric Research Utrecht (IMAU), Utrecht University, The \\ Netherlands \\ BJORN STEVENS \\ Department of Atmospheric Sciences, University of California Los Angeles, U.S.A.
}

(Received in final form 23 September 2003)

\begin{abstract}
Large-eddy simulations of a clear convective boundary layer (CBL) and a stratocumulustopped boundary layer are studied. Bottom-up and a top-down scalars were included in the simulations, and the principle of linear superposition of variables was applied to reconstruct the fields of any arbitrary conserved variable. This approach allows a systematic analysis of countergradient fluxes as a function of the flux ratio, which is defined as the ratio between the entrainment flux and the surface flux of the conserved quantity. In general, the turbulent flux of an arbitrary conserved quantity is counter to the mean vertical gradient if the heights where the vertical flux and the mean vertical gradient change sign do not coincide. The regime where the flux is countergradient is therefore bounded by the so-called zero-flux and zero-gradient heights. Because the vertical flux changes sign only if the entrainment flux has an opposite sign to the surface flux, countergradient fluxes are predominantly found for negative flux ratios. In the CBL the flux ratio for the virtual potential temperature is, to a good approximation, constant, and equal to -0.2 . Only if the moisture contribution to the virtual potential temperature is negligibly small will the flux ratio for the potential temperature be equal to this value. Otherwise, the flux ratio for the potential temperature can have any arbitrary (negative) value, and, as a consequence, the fluxes for the potential temperature and the virtual potential temperature will be countergradient at different heights. As a practical application of the results, vertical profiles of the countergradient correction term for different entrainment-to-surface-flux ratios are discussed.
\end{abstract}

Keywords: Convective boundary layer, Entrainment flux, Non-local transport, Stratocumulus, Turbulence.

^ E-mail: roode@ phys.uu.nl.

$\dagger$ Deceased 18 January 2002.

Boundary-Layer Meteorology 112: 179-196, 2004.

(C) 2004 Kluwer Academic Publishers. Printed in the Netherlands. 


\section{Introduction}

Turbulent transport plays a major role in changing the thermodynamic state of the atmospheric boundary layer. Since the grid resolution of current general circulation models (GCMs) and numerical weather prediction (NWP) models is generally much larger than the typical turbulent eddy sizes in the boundary layer, the tendencies due to turbulent transport need to be parameterized. In analogy with molecular diffusion, one of the most simple means to model the vertical turbulent flux of an arbitrary quantity $\chi$ is by assuming that the vertical flux $\left(\overline{w^{\prime} \chi^{\prime}}\right)$ is downgradient and satisfies,

$$
\overline{w^{\prime} \chi^{\prime}}=-K_{\chi} \frac{\partial \bar{\chi}}{\partial z} .
$$

In general the eddy diffusion coefficient $K_{\chi}$ is a function of the typical length and velocity scales of the turbulence field and varies with height $z$.

Measurements collected in the clear convective boundary layer (CBL) have shown that for some quantities, for example the potential temperature, the flux transport can be counter to its mean vertical gradient (Lenschow, 1970; Warner, 1971). In that case the downgradient formulation (1) will obviously lead to a wrong sign of the potential temperature flux. To account for this effect, Priestley and Swinbank (1947), Deardorff (1972), Troen and Mahrt (1986) and Holtslag and Moeng (1991) proposed to include a countergradient correction term $\gamma_{\chi}$ in (1), viz.

$$
\overline{w^{\prime} \chi^{\prime}}=-K_{\chi}\left(\frac{\partial \bar{\chi}}{\partial z}-\gamma_{\chi}\right) .
$$

Note that this formulation differs only slightly from the one originally proposed by Ertel (1942), who suggested that the potential temperature flux be constituted of two terms, one proportional to the average lapse rate at the same height, the other not. In a wider sense, the factor $\gamma_{\chi}$ can be regarded as a $K_{\chi}$-adjustment factor, since it includes corrections for all situations with the specified $K_{\chi}$.

Because countergradient fluxes are thought to be indicative of boundary-layer scale eddies, as opposed to small-scale eddies, such fluxes are often called nonlocal fluxes. Using different approaches, Wyngaard and Weil (1991) and De Roode et al. (2000) computed similar analytical expressions for $\gamma_{x}$ that include the vertical velocity skewness as a relevant quantity. An expression for the potential temperature flux presented by Zilitinkevich et al. (1999) involves not only the vertical velocity skewness, but also the variances for the vertical velocity and the potential temperature. Gryanik and Hartmann (2002) stressed that the skewness of the potential temperature is a relevant quantity to consider as well. Holtslag and Moeng (1991) used results from a large-eddy simulation (LES) to empirically derive expressions for the non-local closure term. Stevens (2000b) mathematically analyzed 
(2) to show which values for the non-local correction term lead to physically realistic results. A summary of countergradient expressions proposed in the literature is given by Van Dop and Verver (2001).

For a smoke cloud case that combined radiative cooling at the top with a surface heat flux, Cuijpers and Holtslag (1998) diagnosed countergradient fluxes for some quantities. The smoke cloud was introduced by Bretherton et al. (1999) to study the dynamics in the cloudy boundary layer in a simplified way. As in stratocumulus clouds, the top of the smoke-cloud-topped boundary layer is cooled by longwave radiation. Since there is no liquid water present in the smoke-cloud layer, latent heat release effects are effectively switched off. Since the smoke cloud can be considered as an intermediate case between the clear CBL and stratocumulus, the results found by Cuijpers and Holtslag (1998) raise the question whether countergradient fluxes may be present in stratocumulus as well.

It is not clear whether non-local transport in the CBL is a phenomenon that applies to any arbitrary conserved variable. Therefore we aim to study the occurrence of countergradient fluxes for conserved quantities that have different entrainmentto-surface-flux ratios. To this end, a clear convective boundary layer as well as a stratocumulus-topped boundary layer (STBL) will be studied. The latter case is based on observations collected in stratocumulus during the FIRE experiment (Albrecht et al., 1988). For both cases vertical profiles of the countergradient correction term $\gamma_{\chi}$ will be presented for scalars with different entrainment to surface flux ratios.

\section{Experimental Set-Up}

\subsection{Description of the Large-Eddy Simulations}

The results were obtained from the parallelized version of the IMAU/KNMI LES model. The model has been used to study the clear convective boundary layer, and the boundary layer topped by cumulus and stratocumulus (Cuijpers et al., 1996; Siebesma and Cuijpers, 1995; VanZanten et al., 1999). A summary of the model initialization for the CBL and the STBL cases is presented in Table I. The parallel version of the LES model facilitates a simulation on a large horizontal domain, namely $25.6 \times 25.6 \mathrm{~km}^{2}(256 \times 256$ grid points $)$. In the vertical direction the vertical spacing was $20 \mathrm{~m}$ for the CBL and $15 \mathrm{~m}$ for the STBL, and the simulations

lasted $8 \mathrm{hr}$. In this study we will use the LES results from the last hour of the simulation. For this period the velocity scale for the CBL case $w_{*}=1.10 \mathrm{~m} \mathrm{~s}^{-1}$, and the boundary-layer height, $z_{i}=1000 \mathrm{~m}$, and for the stratocumulus case, $w_{*}=$ $0.65 \mathrm{~m} \mathrm{~s}^{-1}$ and $z_{i}=570 \mathrm{~m}$, where (Deardorff, 1980)

$$
w_{*} \equiv\left(2.5 \frac{g}{\theta_{0}} \int_{0}^{z_{i}} \overline{w^{\prime} \theta_{v}^{\prime}} d z\right)^{1 / 3}
$$



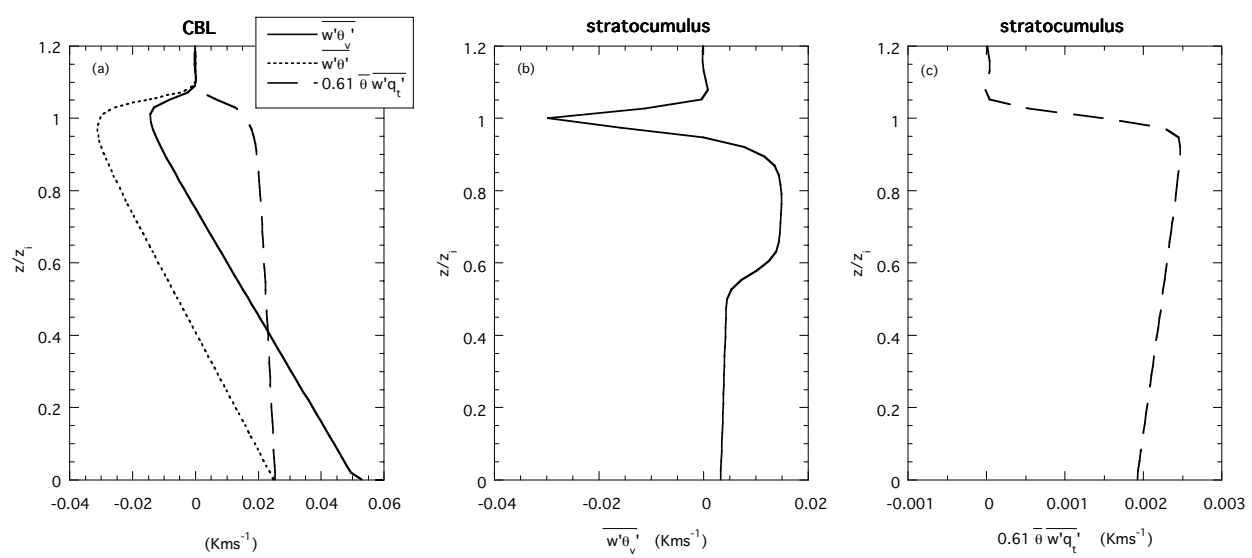

Figure 1. Vertical flux profiles as a function of height during the last hour of the simulation. (a) The virtual potential temperature flux, the potential temperature flux and the specific humidity flux in the CBL. (b) The virtual potential temperature flux and (c) the specific humidity flux in stratocumulus. The specific humidity flux has been multiplied by a factor $0.61 \bar{\theta}$ to show its contribution to the virtual potential temperature flux according to (4). Line styles are according to the legend.

Because of the factor 2.5 , this $w_{*}$ reduces approximately to that defined for the CBL using the surface buoyancy flux.

The surface fluxes in the CBL were set to values such that both the vertical fluxes of the specific humidity $(q)$ and the potential temperature $(\theta)$ contribute $50 \%$ each to the virtual potential temperature flux, which is, to a good approximation, given by

$$
\overline{w^{\prime} \theta_{v}^{\prime}}=\overline{w^{\prime} \theta^{\prime}}+0.61 \bar{\theta} \overline{w^{\prime} q^{\prime}},
$$

where $w$ is the vertical velocity and the virtual potential temperature $\theta_{v}$ is defined as,

$$
\theta_{v}=\theta(1+0.61 q) .
$$

In particular above sea surfaces one can observe such a large contribution of the moisture flux to the buoyancy flux (Durand et al., 2000). As a result of the large moisture flux contribution, the flux profiles for $\theta$ and $\theta_{v}$ differ significantly as shown in Figure 1a.

Observations and LES studies (Stull, 1988; Lewellen and Lewellen, 1998; VanZanten et al., 1999) show that the flux ratio for $\theta_{v}, r_{\theta_{v}}$,

$$
r_{\theta_{v}}=\frac{\overline{w^{\prime} \theta_{v}^{\prime}}}{\overline{w^{\prime} \theta_{v 0}^{\prime}}} \approx-0.2
$$

where the entrainment and surface fluxes are indicated by the subscripts $T$ and 0 , respectively. For an inversion layer that is infinitesimally thin, the flux at the top of the boundary layer can be expressed as (Lilly, 1968)

$$
\overline{w^{\prime} \theta_{v T}^{\prime}}=-w_{e} \Delta \bar{\theta}_{v},
$$




\section{TABLE I}

Summary of the initialization values used for the large-eddy simulation of the convective boundary layer ('CBL') and the nocturnal stratocumulus case. $\theta_{l}$ represents the liquid water potential temperature, $q_{t}$ the total specific humidity, and $u$ and $v$ the horizontal wind components. The subscript 'BL' indicates a constant value within the boundary layer, $\Delta$ the jump across the inversion, 'GEO' the geostrophic wind velocity, $d / d z_{F A}$ the vertical gradient above the inversion in the free atmosphere, and the subscript ' 0 ' the value of the flux at the surface. The variables $\psi$ and $\phi$ represent the bottom-up and top-down scalar, respectively.

\begin{tabular}{|c|c|c|c|}
\hline & CBL & Stratocumulus & Units \\
\hline$p_{\text {surf }}$ & 1040.0 & 1012.5 & {$[\mathrm{hPa}]$} \\
\hline$\frac{d \bar{w}}{d z}$ & 0.0 & $-1 \times 10^{-5}$ & {$\left[\mathrm{~s}^{-1}\right]$} \\
\hline Inversion base & 810.0 & 577.5 & {$[\mathrm{~m}]$} \\
\hline Inversion top & 830.0 & 592.5 & {$[\mathrm{~m}]$} \\
\hline$u_{g e o}$ & 0.001 & 3.4 & {$\left[\mathrm{~m} \mathrm{~s}^{-1}\right]$} \\
\hline$v_{\text {geo }}$ & 0.0 & -4.9 & {$\left[\mathrm{~m} \mathrm{~s}^{-1}\right]$} \\
\hline $\bar{u}_{B L}$ & 0.001 & 3.4 & {$\left[\mathrm{~m} \mathrm{~s}^{-1}\right]$} \\
\hline $\bar{v}_{B L}$ & 0.0 & -4.9 & {$\left[\mathrm{~m} \mathrm{~s}^{-1}\right]$} \\
\hline${\overline{\theta_{l}}}_{B L}$ & 305.0 & 287.5 & {$[\mathrm{~K}]$} \\
\hline${\overline{q_{t}}}_{B L}$ & 16.0 & 9.6 & {$\left[\mathrm{~g} \mathrm{~kg}^{-1}\right]$} \\
\hline $\bar{\psi}_{B L}$ & 1.0 & 1.0 & \\
\hline $\bar{\phi}_{B L}$ & 1.0 & 1.0 & \\
\hline$\Delta \bar{u}$ & 0.0 & 0.0 & {$\left[\mathrm{~m} \mathrm{~s}^{-1}\right]$} \\
\hline$\Delta \bar{v}$ & 0.0 & 0.0 & {$\left[\mathrm{~m} \mathrm{~s}^{-1}\right]$} \\
\hline$\Delta \overline{\theta_{l}}$ & 5.0 & 12.0 & {$[\mathrm{~K}]$} \\
\hline$\Delta \overline{q_{t}}$ & -14.0 & -3.0 & {$\left[\mathrm{~g} \mathrm{~kg}^{-1}\right]$} \\
\hline$\Delta \bar{\psi}$ & 0.0 & 0.0 & \\
\hline$\Delta \bar{\phi}$ & 0.1 & 0.1 & \\
\hline$\left(\frac{d \overline{\theta_{l}}}{d z}\right)_{F A}$ & 12.26 & 7.5 & {$\left[\mathrm{~K} \mathrm{~km}^{-1}\right]$} \\
\hline$\left(\frac{d}{d z}\right)_{F A}$ & 0.0 & -3.0 & {$\left[\left(\mathrm{~g} \mathrm{~kg}^{-1}\right) \mathrm{km}^{-1}\right]$} \\
\hline$w^{\prime} \theta_{l 0}^{\prime}$ & 0.025 & 0.0013 & {$\left[\mathrm{~m} \mathrm{~K} \mathrm{~s}^{-1}\right]$} \\
\hline$\overline{w^{\prime} q_{t 0}^{\prime}}$ & $1.366 \times 10^{-1}$ & $1.09 \times 10^{-2}$ & {$\left[\left(\mathrm{~g} \mathrm{~kg}^{-1}\right)\left(\mathrm{m} \mathrm{s}^{-1}\right)\right]$} \\
\hline$\overline{w^{\prime} \psi^{\prime}} 0$ & 0.001 & 0.001 & {$\left[\mathrm{~m} \mathrm{~s}^{-1}\right]$} \\
\hline$\overline{w^{\prime} \phi^{\prime}}{ }_{0}$ & 0.0 & 0.0 & {$\left[\mathrm{~m} \mathrm{~s}^{-1}\right]$} \\
\hline$\overline{w^{\prime} \theta_{v 0}^{\prime}}$ & 0.053 & 0.0032 & {$\left[\mathrm{~m} \mathrm{~K} \mathrm{~s}^{-1}\right]$} \\
\hline$u_{*}$ & 0.01 & 0.18 & {$\left[\mathrm{~m} \mathrm{~s}^{-1}\right]$} \\
\hline
\end{tabular}


where $\Delta$ represents the difference between the mean values across the inversion. Combination of (6) and (7) yields for the entrainment rate $w_{e}$

$$
w_{e}=-r_{\theta_{v}} \frac{\overline{w^{\prime} \theta_{v 0}^{\prime}}}{\Delta \bar{\theta}_{v}} .
$$

In general, if there is a non-zero moisture flux in the boundary layer the flux ratios for $\theta$ and $\theta_{v}$ will differ. Generic expressions that relate the flux ratio of both $q$ and $\theta$ to the flux ratio of $\theta_{v}$ are (De Roode et al., 2004)

$$
r_{\theta}=r_{\theta_{v}}\left(\frac{1+\frac{0.61 \bar{\theta} \overline{w^{\prime} q^{\prime} 0}}{\overline{w^{\prime} \theta^{\prime}} 0}}{1+\frac{0.61 \bar{\theta} \Delta \bar{q}}{\Delta \bar{\theta}}}\right), r_{q}=r_{\theta_{v}}\left(\frac{1+\frac{\overline{w^{\prime} \theta^{\prime} 0}}{0.61 \bar{\theta} \overline{w^{\prime} q^{\prime}}}}{1+\frac{\Delta \bar{\theta}}{0.61 \bar{\theta} \Delta \bar{q}}}\right) \text {. }
$$

The stratocumulus case is based on observations made off the coast of California during the FIRE field experiment (Hignett, 1991; Duynkerke and Hignett, 1993; Duynkerke and Teixeira, 2001). As part of an intercomparison study in the framework of the European Project on Cloud Systems (EUROCS) the diurnal cycle of this stratocumulus case has been simulated numerically by a variety of different models ranging from single column models to LES models. In the present study we only consider stratocumulus during the night implying that shortwave radiation was not included. The vertical fluxes of the virtual potential temperature and the total specific humidity $\left(q_{t}\right)$ are shown in Figures $1 \mathrm{~b}$ and $\mathrm{c}$. The STBL is vertically well-mixed during the entire simulation, and during the last hour the mean cloud base height is at about $300 \mathrm{~m}$.

\subsection{ANALYSIS PROCEDURE}

We have added two conserved, passive scalars to the simulations, $\psi$ and $\phi$, respectively. We define conserved variables as quantities that are conserved under adiabatic processes, regardless of the state of the saturation of the air parcel (Stull, 1988). To diagnose the fields of an arbitrary passive scalar $\chi$ we will make use of the principle of superposition of variables (Wyngaard and Brost, 1984; Jonker et al., 1999). The quantity $\chi$ is given by a linear superposition of two variables $\psi$ and $\phi$, and an arbitrary constant $c$,

$$
\chi=a \psi+b \phi+c .
$$

The variable $\psi$ has a non-zero flux at the surface, and is therefore referred to as a 'bottom-up' scalar. In contrast, the 'top-down' scalar $\phi$ has no surface flux. However, a turbulent flux is generated by entrainment at the top of the boundary layer caused by a jump $\Delta \bar{\psi}$ across the inversion, analogously to (7). Note that we have multiplied the bottom-up and top-down fields with a constant, such that their fluxes are set to unity, $\overline{w^{\prime} \psi^{\prime}}{ }_{0}=1 \mathrm{~m} \mathrm{~s}^{-1}$ and $\overline{w^{\prime} \phi^{\prime}}{ }_{T}=1 \mathrm{~m} \mathrm{~s}^{-1}$. In the remainder 


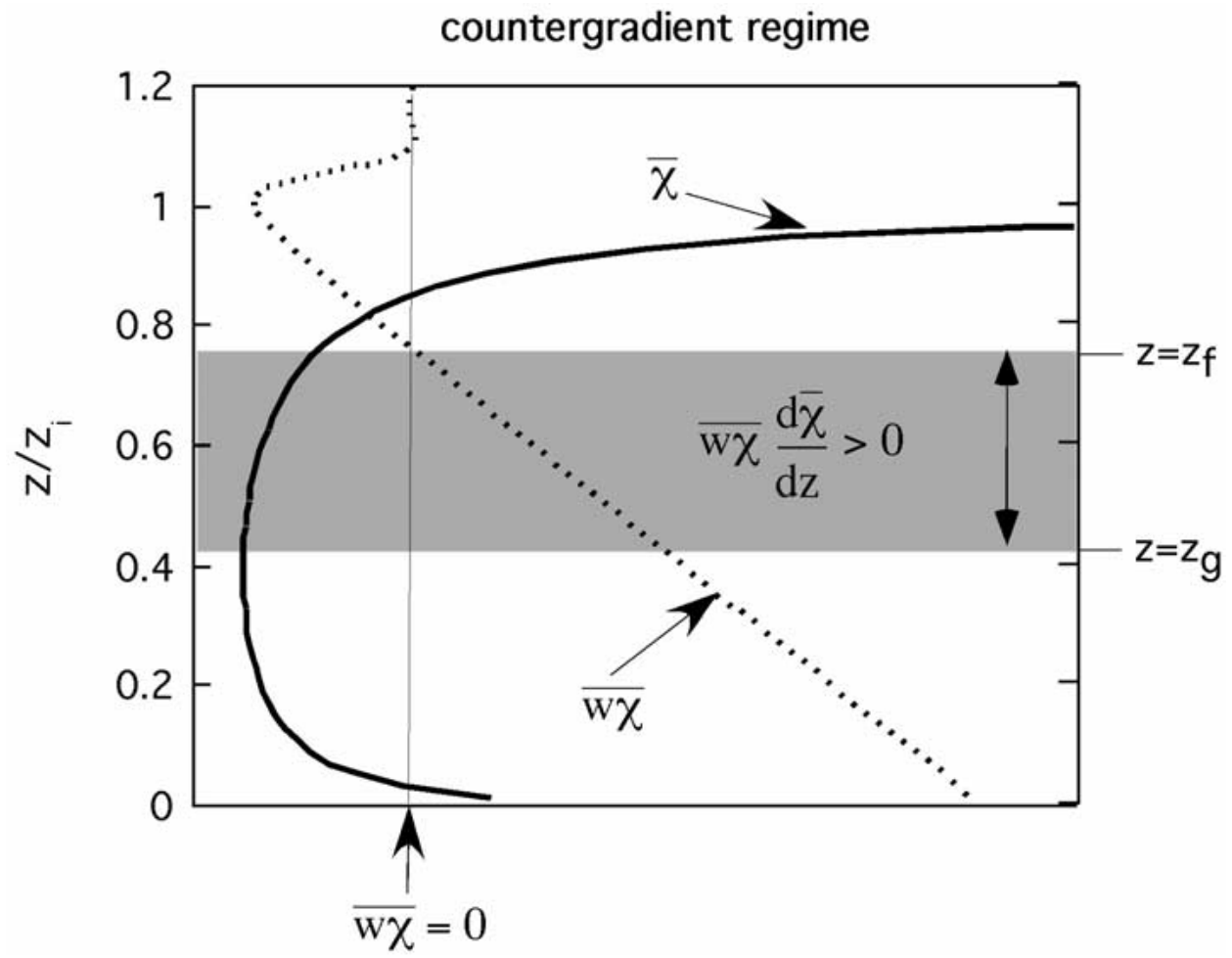

Figure 2. Schematic showing the vertical profiles of the mean $(\bar{\chi})$ and the vertical flux $\left(\overline{w^{\prime} \chi^{\prime}}\right)$ of a quantity with a flux ratio $r_{\chi}=-0.25$. The countergradient flux regime is bounded by the 'zero-flux' height $z_{f}$ and the 'zero-gradient' height $z_{g}$.

of the paper we will use these fields. Vertical profiles of the bottom-up and topdown fluxes are displayed in Figure 3. Note that the fluxes exhibit some curvature effects and deviate slightly from a linear profile because the boundary-layer height $z_{i}$ grows with time, i.e., $d z_{i} / d t \neq 0$ (Wyngaard and Brost, 1984).

After applying Reynolds decomposition on (10) and multiplication by $w^{\prime}$ we can express the vertical flux $\overline{w^{\prime} \chi^{\prime}}$ as a function of the bottom-up and top-down fluxes,

$$
\overline{w^{\prime} \chi^{\prime}}=a \overline{w^{\prime} \psi^{\prime}}+b \overline{w^{\prime} \phi^{\prime}}
$$

By choosing appropriate values for $a$ and $b$ we can obtain any arbitrary flux ratio $r_{\chi}$,

$$
r_{\chi}=\frac{\overline{w^{\prime} \chi^{\prime}} T}{\overline{w^{\prime} \chi_{0}^{\prime}}}=\frac{b}{a}
$$


Vertical fluxes in the boundary layer will be counter to the mean vertical gradient if the following criterion is satisfied,

$$
\overline{w^{\prime} \chi^{\prime}} \frac{\partial \bar{\chi}}{\partial z}>0 .
$$

Figure 2 shows an example of a countergradient flux layer for $r_{\chi}=-0.25$. A layer with countergradient fluxes for a quantity $\chi$ with flux ratio $r_{\chi}$ exists, if there is a level $z_{f}<z_{i}$ where the vertical flux vanishes,

$$
\left.\overline{w^{\prime} \chi^{\prime}}\right|_{z=z_{f}}=0 \text {, }
$$

and which does not coincide with the height $z_{g}$ where its mean vertical gradient equals zero,

$$
\left.\frac{\partial \bar{\chi}}{\partial z}\right|_{z=z_{g}}=0 .
$$

Note that by (10)-(12), the criterion for countergradient fluxes (13) is equivalent to

$$
\left(\overline{w^{\prime} \psi^{\prime}}+r_{\chi} \overline{w^{\prime} \phi^{\prime}}\right)\left(\frac{\partial \bar{\psi}}{\partial z}+r_{\chi} \frac{\partial \bar{\phi}}{\partial z}\right)>0 .
$$

Thus, the heights where (13) is satisfied can be obtained by applying (16) for any arbitrary flux ratio $r_{\chi}$. Therefore, the results shown in Figures 3 and 4 are the basic ingredients needed to diagnose the countergradient flux regime.

The zero-flux level can also be computed analytically, provided that the boundary layer is in a quasi-steady state, meaning that the shape of the vertical profile for the mean of $\chi$ is stationary. In that case its vertical flux is linear,

$$
\frac{\overline{w^{\prime} \chi^{\prime}}(z)}{\overline{w^{\prime} \chi_{0}^{\prime}}}=\left(1-z / z_{i}\right)+r_{\chi} z / z_{i},
$$

and the height $z_{f}$ where $\left.\overline{w^{\prime} \chi^{\prime}}\right|_{z=z_{f}}=0$ is given by

$$
z_{f}\left(r_{\chi}\right) z_{i}=\frac{1}{1-r_{\chi}}
$$

Because $z_{f}<z_{i}$, it follows from this expression that $r_{\chi}<0$. Thus one of the boundaries of the countergradient flux regime is given by the level where the mean vertical flux changes sign, which implies that the entrainment and surface flux of $\chi$ must have an opposite sign. 


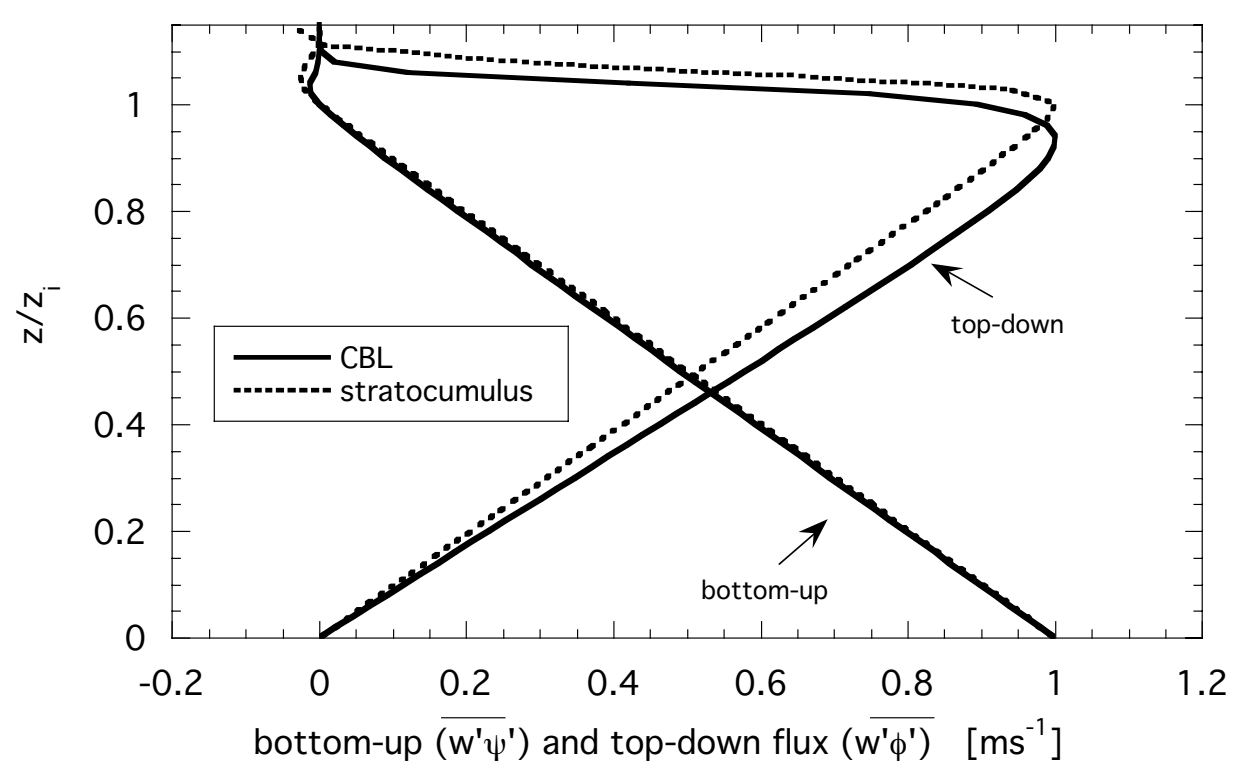

Figure 3. Vertical flux profiles of the bottom-up $\left(\overline{w^{\prime} \psi^{\prime}}\right)$ and top-down $\left(\overline{w^{\prime} \phi^{\prime}}\right)$ scalar as a function of height in the CBL and in stratocumulus. The fluxes represent horizontal slab-average values over the last hour of simulation. Line styles are according to the legend.

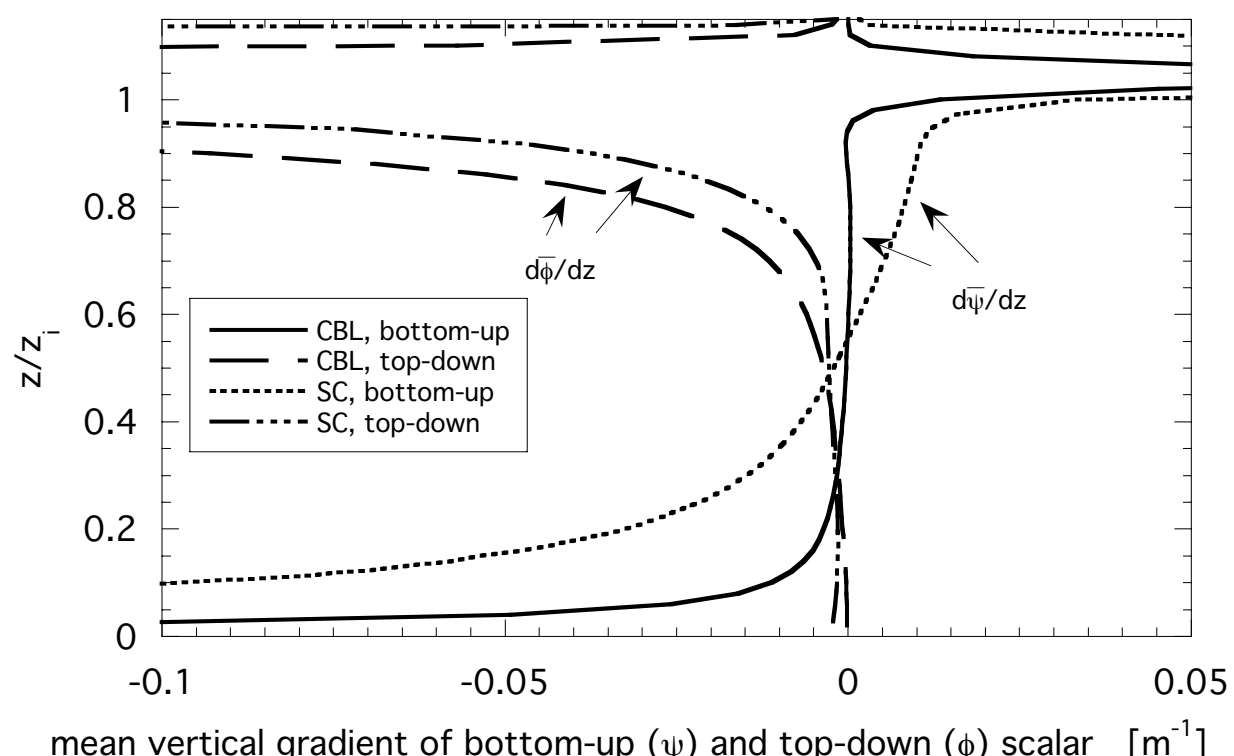

Figure 4. Mean vertical gradients for the bottom-up $(\psi)$ and top-down $(\phi)$ scalar in the CBL and in stratocumulus ('SC'). The gradients represent represent horizontal slab-average values over the last hour of simulation. Line styles are according to the legend. 


\section{The Countergradient Regime}

\section{1. $\mathrm{CBL}$}

Figure 5 shows that in the CBL countergradient fluxes are manifest for negative flux ratios only. Indeed, it appears that the zero-flux level (i.e., where $\overline{w^{\prime} \chi^{\prime}}=0$ ) is a good predictor for the levels were one can expect countergradient fluxes. Obviously, there is no symmetry at $r_{\chi}=0$. For quantities that have a very small positive flux ratio $\left(r_{\chi}<<1\right)$, the flux is countergradient in the upper part of the boundary layer, although from the figure this is hard to distinguish by the eye. This finding is consistent with the LES results reported by Wyngaard and Brost (1984). For all other quantities that have a positive vertical flux throughout the boundary layer a correction term that accounts for countergradient behaviour like $\gamma_{x}$ in (2) is not necessary. This is for example the case for the moisture flux, provided that the specific humidity above the inversion is lower than in the boundary layer. The fluxes for the virtual potential temperature and the potential temperature are counter to their mean vertical gradients at different heights, which is due to their different flux ratios.

It should be emphasized at this point that the shape of the diagram displayed in Figure 5 does not depend on the values for the fluxes of moisture and potential temperature. The flux ratio for the virtual potential temperature has, to a good approximation, a constant value. If one would repeat the same CBL simulation with other surface fluxes and inversion jumps for $q$ and $\theta$, but with the same virtual potential temperature inversion jump and surface flux $\overline{w^{\prime} \theta_{v 0}^{\prime}}$, the passive scalars would be advected in an identical way since the dynamics are fully determined by the virtual potential temperature flux. Because moisture and potential temperature appear to control the virtual potential temperature by its definition (5), one would be tempted to refer to $q$ and $\theta$ as dynamic quantities. However, the fields of the latter two do not behave in another way as passive scalars. To put it even stronger, the field of a passive scalar with a flux ratio $r_{\chi}=r_{\theta}$ is equal to the field of $\theta$ up to a constant and a linear factor; this implies that the correlation coefficient between the fields $\chi$ and $\theta$ is equal to 1 . Consequently, given $r_{\theta}$, the heights where $\overline{w^{\prime} \theta^{\prime}}$ is counter to the mean vertical gradient are predicted by Figure 5. It is interesting to note that there is a direct analogy with the ocean mixed layer where observations show that the temperature and salinity gradients on horizontal scales of 20 metres to 10 kilometres tend to compensate their effect on the density (Rudnick and Ferrari, 1999), as is the case for the moisture and the potential temperature in their effect on the virtual potential temperature at the mesoscales of this CBL (De Roode et al., 2004). 
CBL

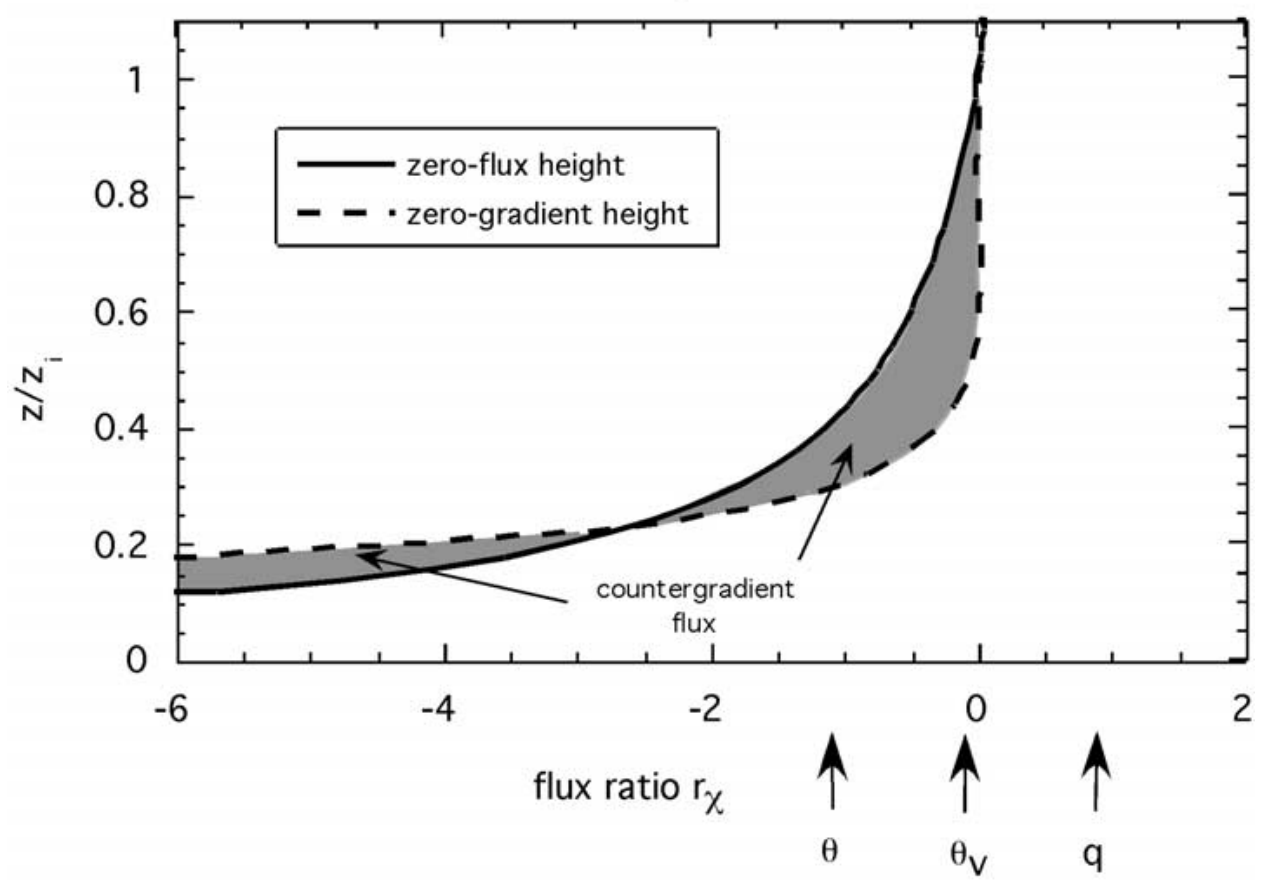

Figure 5. The grey-shaded area indicates the levels where the mean vertical turbulent flux of $\chi$ is counter the mean vertical gradient of $\chi$ in the CBL. The height is scaled by the boundary-layer depth $z_{i}$. The thick solid line represents the level where the vertical flux is zero, $\overline{w^{\prime} \chi^{\prime}}=0$ ('zero-flux height'), and the thick dashed line indicates the level where the mean vertical gradient is zero, $\partial \bar{\chi} / \partial z=0$ ('zero-gradient height'). The vertically pointing arrows indicate the flux ratios for the potential temperature $\theta$, virtual potential temperature $\theta_{v}$ and the specific humidity $q$.

\subsection{Stratocumulus}

Figure 6 shows that stratocumulus also exhibit a countergradient regime, which appears qualitatively similar to the one for the CBL. However, for the stratocumulus case there is also a limited range of positive flux ratios where the flux is countergradient, despite the fact that the vertical flux does not change sign. For these instances the mean vertical gradient changes sign twice within the boundary layer.

The picture that emerges from this study is that in the atmospheric CBL a layer with countergradient fluxes exists, since the zero-flux and zero-gradient heights generally do not coincide. Because the zero-flux height is associated with negative flux ratios there is an asymmetry around $r_{\chi}=0$, which implies that countergradient fluxes can be expected to occur predominantly for situations where the surface flux has an opposite sign to the entrainment flux. 
While the countergradient regime for the CBL is universal as long as the flux ratio for the buoyancy $r_{\theta_{v}} \approx-0.2$, this is not the case for the stratocumulus countergradient regime displayed in Figure 6. The turbulence field in stratocumulus does not scale universally because the buoyancy flux, which is the primary production source for turbulent kinetic energy, has typically a non-linear vertical profile due to radiative and latent heat release effects as cloud liquid water droplets condensate or evaporate (Stevens, 2000a; Nicholls, 1984). Latent heat release effects partly explain the enhancement of the buoyancy flux in the cloud layer (see Figure 1b), whereas the jump at the top of the cloud is due to a longwave radiative cooling rate. The local minimum of the buoyancy flux at the top of the boundary layer is due to entrainment of relatively warm and dry air from just above the inversion. The value for the minimum buoyancy flux at the boundary-layer top is proportional to the entrainment rate, which, in turn, is dependent on the buoyancy production rate of turbulent kinetic energy in the boundary layer. Thus there is an implicit relation between the entrainment rate and the buoyancy flux profile, which is not yet fully understood (Stevens, 2002). Nonetheless, although the precise location of the zero-gradient height for stratocumulus will in general depend on the detailed structure of the turbulence field, it is not very likely that it will coincide with the zero-flux height for all (negative) flux ratios, implying that countergradient fluxes are a characteristic feature of boundary-layer convection.

\section{The Countergradient Correction Term $\gamma_{\chi}$}

Holtslag and Moeng (1991) showed that in the interior of the CBL $\gamma_{\theta_{v}}$ can be well approximated by a constant value. To assess whether this value is appropriate for quantities that have a different flux ratio, we will apply the linear superposition of variables to diagnose vertical profiles of $\gamma_{\chi}$ for different flux ratios $r_{\chi}$.

In the following, we will use non-dimensional variables (indicated by hats),

$$
\begin{aligned}
& \hat{z} \equiv z / z_{i}, \\
& \hat{K}_{\chi} \equiv K_{\chi} /\left(w_{*} z_{i}\right), \\
& \hat{\gamma}_{\chi} \equiv \gamma_{\chi} z_{i} / \chi_{*}, \\
& (\hat{\chi}, \hat{\psi}, \hat{\phi}) \equiv\left(\bar{\chi} / \chi_{*}, \bar{\psi} / \psi_{*}, \bar{\phi} / \phi_{*}\right),
\end{aligned}
$$

where

$$
\left(\chi_{*}, \psi_{*}, \phi_{*}\right) \equiv\left(\overline{w^{\prime} \chi^{\prime}}, \overline{w^{\prime} \psi^{\prime}}, \overline{w^{\prime} \phi^{\prime}}{ }_{T}\right) / w_{*} .
$$

Note that we set $\overline{w^{\prime} \chi^{\prime}}{ }_{0}=1 \mathrm{~m} \mathrm{~s}^{-1}$, such that $\chi_{*}=\psi_{*}=\phi_{*}$. Furthermore, we will assume that in a quasi-stationary situation the flux profiles are linear. Substitution 
stratocumulus

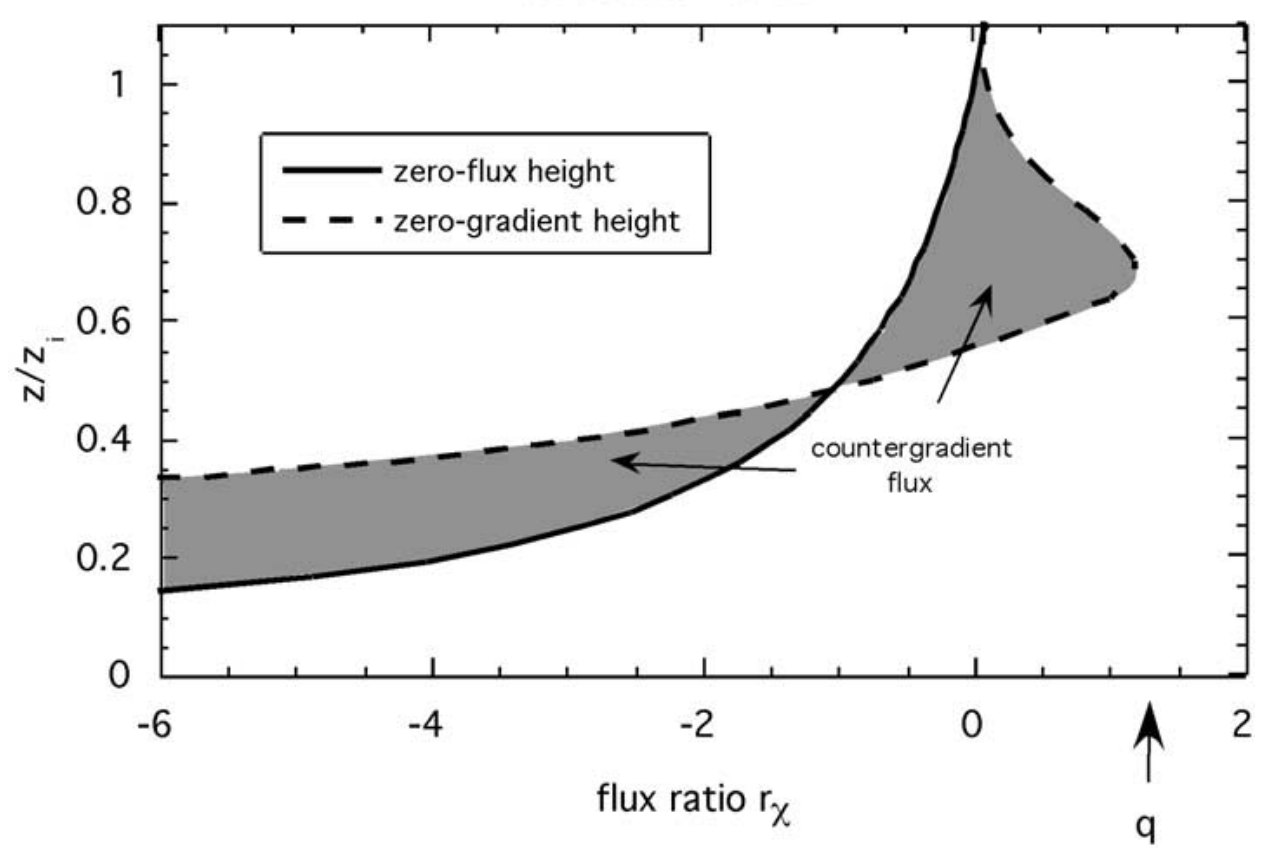

Figure 6. As in Figure 5 except for stratocumulus. The vertically pointing arrow indicates the flux ratio for the specific humidity $q$. Due to the radiative cooling at the top, temperature variables are not conserved and their flux ratios are therefore not indicated in this diagram.

of (17) into (2), and applying the linear superposition of variables (10), gives the following expression for the non-dimensional countergradient factor $\hat{\gamma}_{x}$

$$
\hat{\gamma}_{\chi}=\frac{1-\hat{z}+r_{\chi} \hat{z}}{\hat{K}_{\chi}}+\left(\frac{\partial \hat{\psi}}{\partial \hat{z}}+r_{\chi} \frac{\partial \hat{\phi}}{\partial \hat{z}}\right) .
$$

Equation (24) provides the vertical profile of $\hat{\gamma}_{x}$ for any arbitrary flux ratio and eddy-diffusivity coefficient on the basis of just two variables, namely the vertical gradients of the bottom-up and top-down scalars. To diagnose $\hat{\gamma}_{x}(z)$ for different flux ratios, we will use the following vertical profile for the eddy diffusivity $\hat{K}_{\chi}$,

$$
\hat{K}_{\chi}(\hat{z})=k \hat{z}(1-\hat{z})^{2},
$$

with $k=0.675$. This $\hat{K}_{\chi}$ profile was used in the study of Stevens (2000b), and was derived on the basis of the vertical velocity variance and mean virtual potential temperature vertical profiles in the CBL.

Figure 7a shows that in the bulk of the CBL the $\hat{\gamma}_{\chi}$ profile for $r_{\chi}=-0.25$, which corresponds approximately to the flux ratio for the virtual potential temperature, can be well approximated by a constant value, $\hat{\gamma}_{x}=5$. However, for other 

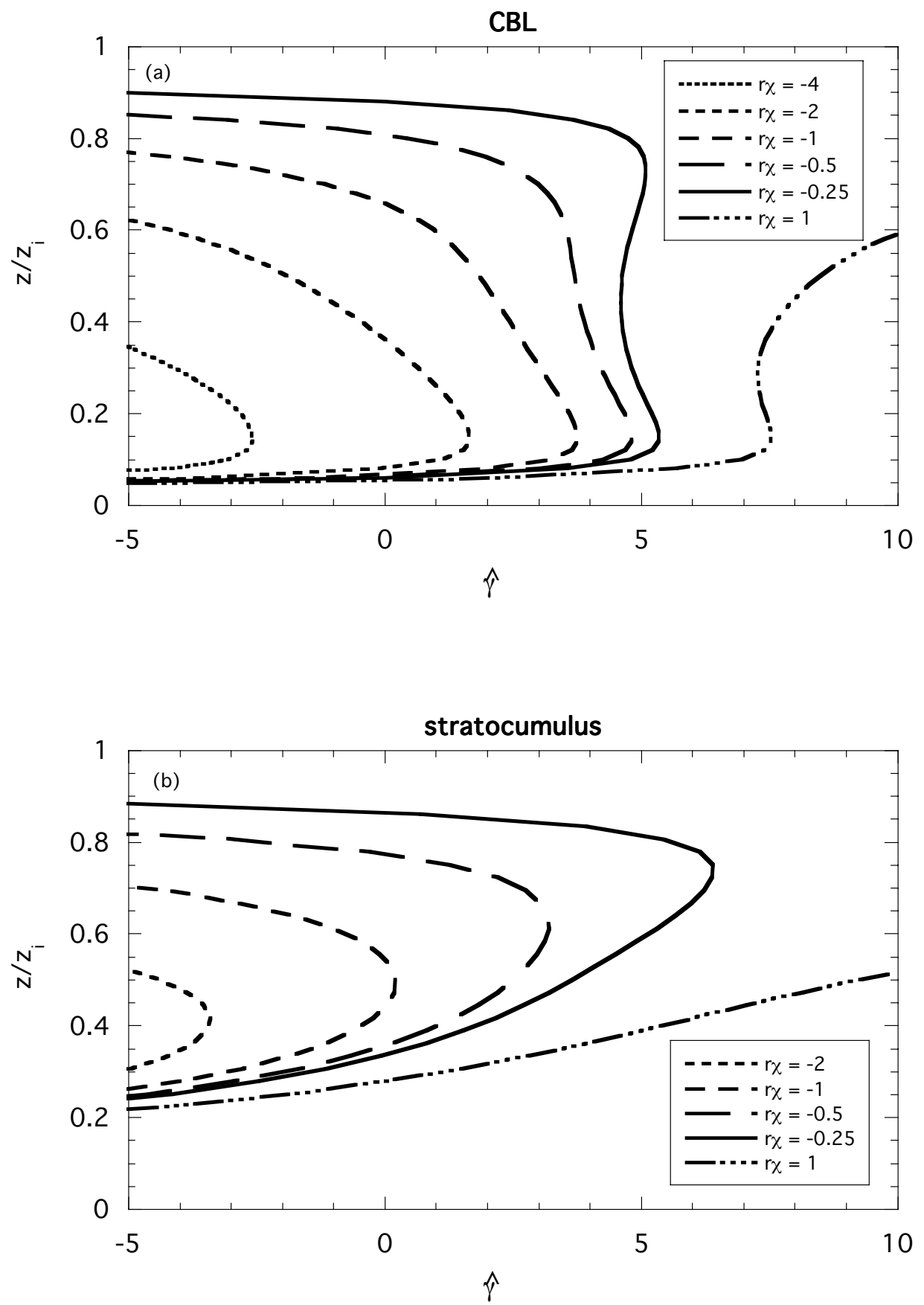

Figure 7. Vertical profiles of the non-dimensionalized countergradient factor $\hat{\gamma}_{\chi}$ for different flux ratios $r_{\chi}$. (a) The CBL and (b) stratocumulus. The line styles are according to the legend. 
flux ratios $\hat{\gamma}_{x}$ varies considerably with height. This is also the case for the flux ratios presented for the stratocumulus case. At the top of both boundary layers, $\left|\hat{\gamma}_{\chi}\right|$ increases for increasing $\left|r_{\chi}\right|$. This behaviour can be understood from the structure of Equation (24), which predicts that for $\left|r_{\chi}\right|>>1$,

$$
\hat{\gamma}_{\chi}=r_{\chi}\left(\frac{\hat{z}}{\hat{K}_{\chi}}+\frac{\partial \hat{\phi}}{\partial \hat{z}}\right) .
$$

Noting that the vertical gradient of the top-down scalar is maximum at the top of the boundary layer, a likely physical interpretation of this solution is that for large absolute values of the flux ratio the countergradient correction term must represent the effect of the entrainment flux at the boundary-layer top. Lastly, it should be remarked that the countergradient correction term does not vanish for quantities whose flux is downgradient at all heights, as is clear from the examples shown for $r_{\chi}=1$.

In summary, our diagnosis suggests that a fixed eddy-diffusivity profile based on the mean virtual potential temperature vertical profile is not appropriate for scalars that have a different flux ratio. Moreover, our findings suggest that eddy diffusivity profiles should be designed such that they better take into account the effect of entrainment. We believe that the application of the principle of linear superposition of variables is a helpful tool for this purpose, since the fields of just one bottom-up and one top-down scalar are sufficient to reconstruct the fields of any arbitrary conserved variable. This approach therefore facilitates the validation of a parameterization for the widest range of flux ratios.

\section{Discussion}

Large-eddy simulations of a clear convective boundary layer and a stratocumulustopped boundary layer are used to investigate for which entrainment-to-surfaceflux ratio the mean vertical flux of an arbitrary conserved quantity is counter to the mean vertical gradient. In general, a layer with countergradient fluxes is bounded by the level where the vertical flux of a quantity $\chi$ changes sign and the level where the vertical gradient of its mean value vanishes. Typically, these levels do not coincide. By means of a linear superposition of the top-down and the bottom-up scalar fields we have computed the zero-flux and zero-gradient levels as a function of the entrainment to surface flux ratio. Since the zero-flux height is associated with a vertical turbulent flux that changes sign somewhere within the boundary layer, the regime where the flux is counter to the mean vertical gradient of the quantity is particularly manifest for negative entrainment to surface flux ratios. In the CBL, the depth of the layer where countergradient fluxes can be expected is most significant for quantities that have a flux ratio around $r_{\chi}=-0.25$, which is close to the 
flux ratio for buoyancy. As a consequence, countergradient flux corrections for quantities that have a positive flux ratio, like moisture, are not necessary.

A good qualitative correspondence between the countergradient regimes for the CBL and stratocumulus is found. However, for stratocumulus countergradient fluxes are also found for a limited range of positive flux ratios despite the fact that the vertical flux does not change sign. In this case the mean vertical gradient changes sign twice within the boundary layer.

In the clear CBL discussed herein the moisture flux contributes significantly to the virtual potential temperature flux. The flux ratio for the potential temperature is $r_{\theta} \approx-1.2$. The larger the contribution of the moisture flux to the buoyancy flux, the more the buoyancy and the potential temperature fluxes will deviate. For parameterizations like (2) this means that in general the buoyancy and the potential temperature fluxes need a different treatment.

Lastly, it is worth noting that countergradient fluxes have an important physical meaning. In the prognostic variance equation,

$$
\frac{\partial \overline{\chi^{\prime 2}}}{\partial t}=-2 \overline{w^{\prime} \chi^{\prime}} \frac{\partial \bar{\chi}}{\partial z}-\frac{\partial \overline{w^{\prime} \chi^{\prime} \chi^{\prime}}}{\partial z}-2 \epsilon_{\chi}
$$

the production term is given by the first term on the right-hand side of (27), and $\epsilon_{\chi}$ represents the dissipation of variance. Therefore, in cases where the flux is counter to the mean vertical gradient the production term becomes negative and will act to consume variance. From this perspective it is interesting to note that studies by Jonker et al. (1999) and De Roode et al. (2004) showed that quantities in the CBL that have a flux ratio close to -0.25 have length scales that are on the order of the boundary-layer depth, whereas quantities that have a flux ratio that significantly deviates from this number the length scales were found to be much larger.

\section{Acknowledgements}

The investigations were supported by the Netherlands Organization for Scientific Research (NWO). This work was sponsored by the National Computing Facilities Foundation (NCF) for the use of supercomputer facilities. Dr. Duynkerke received financial support from the European Project on Cloud Systems (EUROCS), contract EVK2-CT-1999-00051.

\section{References}

Albrecht, B. A., Randall, D. A., and Nicholls, S.: 1988, 'Observations of Marine Stratocumulus Clouds during FIRE', Bull. Amer. Meteorol. Soc. 69, 618-626.

Bretherton, C. S., MacVean, M. K., Bechtold, P., Chlond, A., Cotton, W. R., Cuxart, J., Cuijpers, J. W. M., Khairoutdinov, M., Kosovic, B., Lewellen, D. C., Moeng, C. H., Siebesma, A. 
P., Stevens, B., Stevens, D. E., Sykes, I., and Wyant, M. C.: 1999, 'An Intercomparison of Radiatively-Driven Entrainment and Turbulence in a Smoke Cloud, as Simulated by Different Numerical Models', Quart. J. Roy. Meteorol. Soc. 54, 148-167.

Cuijpers, J. W. M. and Holtslag, A. A. M.: 1998, 'Impact of Skewness and Nonlocal Effects on Scalar and Buoyancy Fluxes in Convective Boundary Layers', J. Atmos. Sci. 55, 151-162.

Cuijpers, J. W. M., Duynkerke, P. G., and Nieuwstadt, F. T. M.: 1996, 'Analyses of Variance and Flux Budgets in Cumulus-Topped Boundary Layers', Atmos. Res. 40, 307-337.

Deardorff, J. W.: 1972, 'Theoretical Expression for the Countergradient Vertical Heat Flux', J. Geophys. Res. 77, 5900-5904.

Deardorff, J. W.: 1980, 'Cloud-Top Entrainment Instability’, J. Atmos. Sci. 37, 131-147.

De Roode, S. R., Duynkerke, P. G., and Jonker, H. J. J.: 2004, 'Large Eddy Simulation: How Large Is Large Enough?', J. Atmos. Sci. 61, 403-421.

De Roode, S. R., Duynkerke, P. G., and Siebesma, A. P.: 2000, 'Analogies between Mass-Flux and Reynolds-Averaged Equations', J. Atmos. Sci. 57, 1585-1598.

Durand, P., Thoumieux, F., and Lambert, D.: 2000, 'Turbulent Length-Scales in the Marine Atmospheric Mixed Layer', Quart. J. Roy. Meteorol. Soc. 126, 1889-1912.

Duynkerke, P. G. and Hignett, P.: 1993, 'Simulation of Diurnal Variation in a Stratocumulus-Capped Marine Boundary Layer during FIRE', Mon. Wea. Rev. 121, 3291-3300.

Duynkerke, P. G. and Teixeira, J.: 2001, 'Comparison of the ECMWF Reanalysis with FIRE I Observations: Diurnal Variation of Marine Stratocumulus', J. Climate 14, 1466-1478.

Ertel, H.: 1942, 'Der vertikale Turbulenz-Wärmestrom in der Atmosphäre', Meteorol. Z. 59, 250253.

Gryanik, V. M. and Hartmann, J.: 2002, 'A Turbulence Closure for the Convective Boundary Layer Based on a Two-Scale Mass-Flux Approach', J. Atmos. Sci. 59, 2729-2744.

Hignett, P.: 1991, 'Observations of the Diurnal Variation in a Cloud-Capped Marine Boundary Layer', J. Atmos. Sci. 48, 1474-1482.

Holtslag, A. A. M. and Moeng, C. H.: 1991, 'Eddy Diffusivity and Countergradient Transport in the Convective Atmospheric Boundary Layer', J. Atmos. Sci. 48, 1690-1698.

Jonker, H. J. J., Duynkerke, P. G., and Cuijpers, J. W. M.: 1999, 'Mesoscale Fluctuations in Scalars Generated by Boundary Layer Convection', J. Atmos. Sci. 56, 801-808.

Lenschow, D. H.: 1970, 'Airplane Measurements of Planetary Boundary Layer Structure', J. Appl. Meteorol. 9, 874-884.

Lewellen, D. C. and Lewellen, W. S.: 1998, 'Large Eddy Boundary Layer Entrainment', J. Atmos. Sci. 55, 2645-2665.

Lilly, D.: 1968, 'Models of Cloud-Topped Mixed Layers under a Strong Inversion', Quart. J. Roy. Meteorol. Soc. 94, 292-309.

Nicholls, S.: 1984, 'The Dynamics of Stratocumulus: Aircraft Observations and Comparisons with a Mixed Layer Model', Quart. J. Roy. Meteorol. Soc. 110, 783-820.

Priestley, C. H. B. and Swinbank, W. C.: 1947, 'Vertical Transport of Heat by Turbulence in the Atmosphere', Proc. Roy. Soc. London, pp. 543-561.

Rudnick, D. L. and Ferrari, R.: 1999, 'Compensation of Horizontal Temperature and Salinity Gradients in the Ocean Mixed Layer', Science 283, 526-529.

Siebesma, A. P. and Cuijpers, J. W. M.: 1995, 'Evaluation of Parametric Assumptions for Shallow Cumulus Convection', J. Atmos. Sci. 52, 650-666.

Stevens, B.: 2000a, 'Cloud Transitions and Decoupling in Shear-Free Stratocumulus-Topped Boundary Layers', Geophys. Res. Lett. 27, 2557-2560.

Stevens, B.: 2000b, 'Quasi-Steady Analysis of a PBL Model with an Eddy-Diffusivity Profile and Nonlocal Fluxes', Mon. Wea. Rev. 128, 824-836.

Stevens, B.: 2002, 'Entrainment in Stratocumulus-Topped Mixed Layers', Quart. J. Roy. Meteorol. Soc. 128, 2663-2690. 
Stull, R. B.: 1988, An Introduction to Boundary Layer Meteorology, Kluwer Academic Publishers, Dordrecht, $666 \mathrm{pp}$.

Troen, I. and Mahrt, L.: 1986, 'A Simple Model of the Atmospheric Boundary Layer; Sensitivity to Surface Evaporation', Boundary-Layer Meteorol. 37, 129-148.

Van Dop, H. and Verver, G.: 2001, 'Countergradient Transport Revisited', J. Atmos. Sci. 58, 22402247.

VanZanten, M. C., Duynkerke, P. G., and Cuijpers, J. W. M.: 1999, 'Entrainment Parameterization in Convective Boundary Layers', J. Atmos. Sci. 56, 813-828.

Warner, J.: 1971, 'Observations of the Eddy Fluxes of Heat and Vapour over the Sea', Quart. J. Roy. Meteorol. Soc. 97, 540-547.

Wyngaard, J. C. and Brost, R. A.: 1984, 'Top-Down and Bottom-Up Diffusion of a Scalar in the Convective Boundary Layer', J. Atmos. Sci. 41, 102-112.

Wyngaard, J. C. and Weil, J. C.: 1991, 'Transport Asymmetry in Skewed Turbulence', Phys. Fluids A 3, 155-162.

Zilitinkevich, S., Gryanik, V. M., Lykossov, V. N., and Mironov, D. V.: 1999, 'Third-Order Transport and Nonlocal Turbulence Closures for Convective Boundary Layers', J. Atmos. Sci. 56, 34633477. 